\title{
Prevalence and associated risk factors of malaria among adults in East Shewa Zone of Oromia Regional State, Ethiopia: a cross-sectional study
}

Frew Tadesse ${ }^{1 *}$, Andrew W. Fogarty ${ }^{2}$ and Wakgari Deressa ${ }^{3}$

\begin{abstract}
Background: Malaria is one of the most important causes of morbidity and mortality in sub-Saharan Africa. The disease is prevalent in over 75\% of the country's area making it the leading public health problems in the country. Information on the prevalence of malaria and its associated factors is vital to focus and improve malaria interventions.

Methods: A cross-sectional study was carried out from October to November 2012 in East Shewa zone of Oromia Regional State, Ethiopia. Adults aged 16 or more years with suspected malaria attending five health centers were eligible for the study. Logistic regression models were used to examine the effect of each independent variable on risk of subsequent diagnosis of malaria.

Results: Of 810 suspected adult malaria patients who participated in the study, 204 (25\%) had microscopically confirmed malaria parasites. The dominant Plasmodium species were P. vivax (54\%) and P. falciparum (45\%), with mixed infection of both species in one patient. A positive microscopic result was significantly associated with being in the age group of 16 to 24 years [Adjusted Odds Ratio aOR 6.7; 95\% Cl: 2.3 to 19.5], 25 to 34 years [aOR 4.2; 95\% Cl: 1.4 to 12.4], and 35 to 44 years [aOR 3.7; 95\% Cl: 1.2-11.4] compared to 45 years or older; being treated at Meki health center [aOR 4.1; 95\% Cl: 2.4 to 7.1], being in Shashemene health center [aOR $=2.3 ; 95 \% \mathrm{Cl}: 1.5$ to 4.5 ], and living in a rural area compared to an urban area [aOR 1.7; 95\% Cl: 1.1 to 2.6)].
\end{abstract}

Conclusion: Malaria is an important public health problem among adults in the study area with a predominance of $P$. vivax and P. falciparum infection. Thus, appropriate health interventions should be implemented to prevent and control the disease.

Keywords: Malaria, Prevalence, Diagnosis, Oromia, Ethiopia

\section{Background}

Ethiopia is one of the malaria-epidemic prone countries in Africa. Malaria is prevalent in over $75 \%$ of the country's area, with $68 \%$ of the total population being at risk [1-3]. The disease was responsible for about $12 \%$ of outpatient consultations and $10 \%$ of health facility admissions, and represents the largest single cause of morbidity $[4,5]$. In 2010, there were more than four million clinical and

\footnotetext{
* Correspondence: fretaabrish@gmail.com

${ }^{1}$ School of Public Health, College of Health Sciences and Medicine, Jigjiga

University, Jigjiga, Ethiopia

Full list of author information is available at the end of the article
}

confirmed malaria cases [6]. Ethiopia is at a high risk of epidemics of malaria due to climate and topography. Broad range of epidemics happen every $5-8$ years in some areas due to climatic fluctuations and drought-related nutritional emergencies [7-9]. P. falciparum and P. vivax are the two predominant malaria parasites in the country, accounting for $60-70 \%$ and $30-40 \%$ of infections, respectively [10], transmitted by inoculation by mosquitos (Anopheles species including Anopheles arabiensis) [9].

As per the National Strategic Plan, the four major intervention strategies that are being applied in the country to combat malaria include early diagnosis and 
prompt treatment, selective vector control that involves the use of indoor residual spraying (IRS) and insecticide treated nets (ITNs) and environmental management [11]. Since 2007, malaria control interventions have been scaled-up and significantly reduced the prevalence of malaria [3]. Nevertheless, malaria is still among the leading causes of outpatient visits and hospital admissions in the country [4]. There is a scarcity of information on the prevalence of malaria among suspected malaria patients attending health facilities and the associated individual and household factors. Such information provides both a measure of the pre-test probability of a positive result as well as geographical and personal risk factors for having a positive diagnosis of infection for patients who present with symptoms of malaria. The World Health Organization (WHO) considers that when the slide positivity rate of all febrile patients with suspected malaria is less than $5 \%$, then this region may be considered as transitioning into a state of preelimination of malaria infection [11].

\section{Methods}

\section{Study setting and population}

This study was conducted from October to November 2012 among adults aged 16 years or above who were attending five health centers in East Shewa zone of Oromia Regional State, Ethiopia. The main aim of the study was to determine the impact of health beliefs on time to presentation [12], and data were also collected on knowledge about malaria. East Shewa zone is malaria endemic area located in the Great Rift Valley in southeast Ethiopia. The climate is regarded as tropical, and this area contains a number of large lakes in a lowland setting. Based on the 2007 national census, East Shewa zone had a total population of 1,356,342; of whom $51 \%$ were men and $49 \%$ women [13]. The zone has three hospitals, 18 health centers and 296 health posts.

Malaria is the third leading cause of outpatient department (OPD) visits (36\%) in East Shewa Zone. The study participants were adult patients aged 16 years or above who presented with malaria symptoms who gave blood for microscopic blood film examination at five health centers (Modjo, Meki, Batu, Bulbula, and Shashemene), each health center representing five woredas (districts). Patients who were mentally retarded, critically ill, or unwilling were excluded from the study.

\section{Study design and data collection}

The study design was a cross-sectional study. Quantitative data were collected using pre-tested structured questionnaires, containing questions on socio-demographic characteristics, and knowledge and perception about malaria, specifically developed and applied for data collection using the local language. The interview took place after a blood sample was drawn by finger prick. The trained laboratory technicians administered the questionnaire after obtaining informed consent from an individual with malaria symptoms. The recruitment of the study participants into the study at each site sequentially continued until the required sample size for each health center was completed.

Blood was collected by experienced laboratory technicians from the finger of patients. Then smears were prepared according to the WHO protocol [14]. Parasitemia and species was determined from thick and thin smear [15], respectively. Microscopic examination of thick films using high power magnification for the presence of parasites and parasite species identification using thin films under $1000 \times$ oil immersion objective was done. A minimum of 100 consecutive fields were counted in the thick blood film before a slide was classified as negative [16].

\section{Statistical analysis}

Data were entered using EPI INFO version 3.5.1 software package (CDC, Atlanta, GA, USA) and analyzed using SPSS version 16 (SPSS, Chicago, IL, USA) (Additional file 1). Initial analysis was done using Chi-squared test and subsequent analysis was performed by logistic regression after adjustment for potential confounding variables presented in Table 4. The data were originally collected for a study of malaria and concern about HIV testing [12] among 810 adults (16 years or above). Hence, there is no formal power calculation as this is a secondary analysis of these data. The original sample size was proportionally allocated to each health center considering the total number of suspected malaria patients tested during the previous three months (June-August, 2012) [12].

\section{Results}

Characteristics of the study participants

Eight hundred thirty eight individuals were approached and a total of 810 (97\%) suspected malaria patients attending the health centers participated in the study, with $59 \%$ of patients from urban areas and $41 \%$ from rural areas. The median age of the patients was 27 years (ranging 16 to 80 ). 35\% of participants had attended school to grade nine or above, while $30 \%$ had had no formal education (Table 1).

\section{Knowledge about malaria}

Seven hundred ninety (97\%) of the patients believed that malaria is a major health problem in the study areas. The most commonly cited malaria symptoms included feeling cold (82\%), headache (76\%), fever (69\%), vomiting (53\%), sweating (48\%), and loss of appetite (49\%) (Table 2). The causes of malaria were reported to be mosquito bite by 759 (94\%) individuals, hunger by 276 (34\%) individuals, eating maize stalk by 199 (25\%) individuals, and eating 
Table 1 Socio-demographic characteristics of the study participants

\begin{tabular}{|c|c|c|c|c|c|c|}
\hline \multirow[t]{2}{*}{ Variables } & \multicolumn{5}{|c|}{ Health center } & \multirow[t]{2}{*}{ Total, $n(\%)$} \\
\hline & Modjo & Meki & Batu & Bulbula & Shashemene & \\
\hline \multicolumn{7}{|l|}{ Residence } \\
\hline Rural & 50 & 58 & 61 & 119 & 46 & $334(41 \%)$ \\
\hline Urban & 119 & 117 & 119 & 28 & 93 & 476 (59\%) \\
\hline \multicolumn{7}{|l|}{ Sex } \\
\hline Female & 70 & 92 & 86 & 81 & 68 & 397 (49\%) \\
\hline Male & 99 & 83 & 94 & 66 & 71 & $413(51 \%)$ \\
\hline \multicolumn{7}{|l|}{ Age } \\
\hline $15-24$ & 78 & 46 & 83 & 48 & 58 & 313 (39\%) \\
\hline $25-34$ & 58 & 80 & 66 & 48 & 47 & 299 (37\%) \\
\hline $35-44$ & 24 & 44 & 24 & 37 & 13 & $142(17 \%)$ \\
\hline$>45$ & 9 & 5 & 7 & 14 & 21 & $56(7 \%)$ \\
\hline \multicolumn{7}{|l|}{ Educational status } \\
\hline No formal education & 33 & 61 & 39 & 76 & 38 & 247 (30\%) \\
\hline Grade 4 & 28 & 33 & 26 & 7 & 9 & $103(13 \%)$ \\
\hline Grade 5-8 & 32 & 35 & 47 & 42 & 17 & $173(21 \%)$ \\
\hline$>$ Grade 8 & 76 & 46 & 68 & 22 & 75 & 287 (35\%) \\
\hline \multicolumn{7}{|l|}{ Marital status } \\
\hline Married & 99 & 103 & 93 & 105 & 60 & 460 (57\%) \\
\hline Single & 70 & 58 & 80 & 41 & 74 & $323(40 \%)$ \\
\hline Others & 0 & 14 & 7 & 1 & 5 & $27(3 \%)$ \\
\hline \multicolumn{7}{|l|}{ Religion } \\
\hline Muslim & 15 & 44 & 84 & 125 & 57 & 325 (40\%) \\
\hline Christian & 154 & 131 & 96 & 22 & 82 & 485 (60\%) \\
\hline \multicolumn{7}{|l|}{ Occupation } \\
\hline Farmer & 35 & 69 & 48 & 85 & 11 & 248 (31\%) \\
\hline House wife & 25 & 30 & 26 & 15 & 24 & $120(15 \%)$ \\
\hline Daily laborer & 27 & 8 & 24 & 2 & 6 & $67(8 \%)$ \\
\hline Gov. employee & 21 & 14 & 16 & 5 & 17 & $73(9 \%)$ \\
\hline NGO employee & 28 & 2 & 16 & 0 & 51 & 97 (12\%) \\
\hline Trader & 5 & 24 & 6 & 4 & 3 & $42(5 \%)$ \\
\hline Student & 28 & 28 & 44 & 36 & 27 & 163 (20\%) \\
\hline \multicolumn{7}{|l|}{ Type of roof } \\
\hline Thatched & 32 & 36 & 34 & 64 & 17 & 183 (23\%) \\
\hline Corrugated iron & 137 & 139 & 146 & 83 & 122 & 627 (77\%) \\
\hline
\end{tabular}

immature sugar cane in 196 (24\%) individuals. 803 (99\%) of the patients believed that malaria is a preventable disease.

\section{Household ownership of ITNs}

Fifty percent of patients with suspected malaria had any mosquito nets/ITNs in their household that can be used while sleeping. Out of those who had mosquito nets/ ITNs 195 (48\%) had two, 146 (36\%) had only one, and 54 (13\%) had three mosquito nets/ITNs. In response to a question asked about the frequency of nights slept under mosquito nets/ITNs in the last fifteen days; 214 (52\%) reported all nights, 121 (30\%) sometimes, and 64 (16\%) none of the nights. 241 (59\%) individuals reported sleeping under mosquito net/ITNs in the night prior to presentation to the health center (Table 2).

Prevalence of malaria parasites in the study population Two hundred four (25\%) individuals in the study population had microscopically confirmed malaria parasites 
Table 2 Malaria knowledge and household ownership of ITNs among the study participants

\begin{tabular}{|c|c|c|c|c|c|c|}
\hline \multirow[t]{2}{*}{ Variables } & \multicolumn{5}{|c|}{ Health center } & \multirow[t]{2}{*}{ Total, $n(\%)$} \\
\hline & Modjo & Meki & Batu & Bulbula & Shashemene & \\
\hline \multicolumn{7}{|l|}{ Symptoms of malaria } \\
\hline Fever & 151 & 141 & 97 & 126 & 46 & $561(69 \%)$ \\
\hline Feeling cold & 143 & 136 & 131 & 138 & 117 & $665(82 \%)$ \\
\hline Headache & 127 & 87 & 125 & 136 & 137 & $612(76 \%)$ \\
\hline Vomiting & 81 & 82 & 105 & 75 & 84 & $427(53 \%)$ \\
\hline Joint pain & 84 & 52 & 56 & 31 & 9 & $232(29 \%)$ \\
\hline Loss of appetite & 114 & 136 & 66 & 53 & 32 & $401(49.5 \%)$ \\
\hline Muscle pain & 69 & 20 & 8 & 10 & 5 & $112(14 \%)$ \\
\hline Nausea & 85 & 47 & 11 & 41 & 50 & 234 (29\%) \\
\hline Sweating & 87 & 134 & 11 & 37 & 118 & $387(48 \%)$ \\
\hline \multicolumn{7}{|l|}{ Malaria is preventable } \\
\hline Yes & 169 & 172 & 177 & 146 & 139 & 803 (99\%) \\
\hline No & 0 & 3 & 3 & 1 & 0 & $7(1 \%)$ \\
\hline \multicolumn{7}{|c|}{ Household ownership of ITNs } \\
\hline Yes & 72 & 82 & 101 & 90 & 62 & $407(50 \%)$ \\
\hline No & 97 & 93 & 79 & 57 & 77 & $403(50 \%)$ \\
\hline \multicolumn{7}{|l|}{ Number of ITNs owned } \\
\hline 1 & 21 & 28 & 48 & 25 & 24 & $146(36 \%)$ \\
\hline 2 & 44 & 40 & 34 & 48 & 29 & $195(48 \%)$ \\
\hline 3 & 7 & 13 & 12 & 15 & 7 & $54(13 \%)$ \\
\hline 4 & 0 & 1 & 6 & 2 & 2 & $11(3 \%)$ \\
\hline \multicolumn{7}{|c|}{ Frequency of night slept under ITNs in the last 15 days } \\
\hline All nights & 39 & 28 & 50 & 56 & 41 & $214(52 \%)$ \\
\hline Sometimes & 24 & 35 & 22 & 19 & 21 & $121(30 \%)$ \\
\hline Only few night & 0 & 1 & 3 & 4 & 0 & $8(2 \%)$ \\
\hline None of the nights & 9 & 18 & 26 & 11 & 0 & $64(16 \%)$ \\
\hline
\end{tabular}

in their blood sample. Among those who had a positive laboratory test result, the dominant Plasmodium species were $P$. vivax 111 (54\%), followed by P. falciparum 92 $(45 \%)$, the remaining one $(0.5 \%)$ showed mixed infections of $P$. falciparum and $P$. vivax (Table 3).

\section{Factors associated with malaria positivity}

Among the potential determinants explored regarding the positivity for malaria age being 16 to 24,25 to 34 , and 35 to 44 years compared to an age of 45 years or more; being in Meki or Shashemene compared to Modjo health centers; living in a rural residence compared to living in an urban area were significantly associated with positive test result for malaria. Compared to those aged 45 years or more, those who were in the age group of 16 to 24 years [Adjusted OR $(\mathrm{aOR})=6.7 ; 95 \% \mathrm{CI}$ ( 2.3 to 19.5)], those who were in the age group of 25 to 34 years [aOR $=4.2 ; 95 \% \mathrm{CI}(1.4$ to 12.4$)$ ], those who were in the age group of 35 to 44 years were more likely to have positive test result for malaria [aOR $=3.7 ; 95 \% \mathrm{CI}$ (1.2 to 11.4)] as compared to those in the age group of above 44 years. Those who were living in rural areas were more likely to have positive test result for malaria [aOR $=1.7 ; 95 \%$ CI $(1.1,2.6)]$ as compared to those who were living in urban area (Table 4 ).

\section{Discussion}

This study provides information regarding the prevalence of a positive diagnosis of malaria and its associated risk factors among adults with suspected malaria in malaria endemic areas located in the Great Rift Valley of southeast Ethiopia. This study has demonstrated that in a population of individuals with malaria symptoms, the prevalence of malaria was $25.2 \%$, of which $P$. vivax and P. falciparum accounts for $54 \%$ and $45 \%$, respectively. The present study depicts that being in the productive age group, living in Meki or Shashemene areas, and living in rural areas are risk factors for malaria infection in this population. 
Table 3 Prevalence of malaria among the study participants

\begin{tabular}{|c|c|c|c|c|}
\hline Variables & No. of patients & No. positive slides (\%) & Positive for P. $f(\%)$ & Positive for P. $v(\%)$ \\
\hline \multicolumn{5}{|l|}{ Health center } \\
\hline Modjo & 169 & $30(18 \%)$ & $16(53 \%)$ & $14(47 \%)$ \\
\hline Meki & 175 & $70(40 \%)$ & $41(59 \%)$ & $29(41 \%)$ \\
\hline Batu & 180 & $43(24 \%)$ & 16 (37\%) & $26(60 \%)$ \\
\hline Bulbula & 147 & 19 (13\%) & $6(32 \%)$ & $13(68 \%)$ \\
\hline Shashemene & 139 & $42(30 \%)$ & $13(31 \%)$ & $29(69 \%)$ \\
\hline \multicolumn{5}{|l|}{ Residence } \\
\hline Rural & 334 & $96(29 \%)$ & 47 (49\%) & $48(50 \%)$ \\
\hline Urban & 476 & $108(23 \%)$ & $45(42 \%)$ & $63(58 \%)$ \\
\hline \multicolumn{5}{|l|}{ Sex } \\
\hline Female & 397 & $95(24 \%)$ & $43(45 \%)$ & $51(54 \%)$ \\
\hline Male & 413 & 109 (26\%) & $49(45 \%)$ & $60(55 \%)$ \\
\hline \multicolumn{5}{|l|}{ Age } \\
\hline $15-24$ & 313 & $93(30 \%)$ & $40(43 \%)$ & $52(56 \%)$ \\
\hline $25-34$ & 299 & $75(25 \%)$ & 37 (49\%) & $38(51 \%)$ \\
\hline $35-44$ & 142 & $32(22 \%)$ & $14(44 \%)$ & $18(56 \%)$ \\
\hline$>45$ & 56 & $4(7 \%)$ & $1(25 \%)$ & $3(75 \%)$ \\
\hline \multicolumn{5}{|l|}{ Type of roof } \\
\hline Thatched & 183 & $56(31 \%)$ & $25(45 \%)$ & $30(54 \%)$ \\
\hline Corrugated iron & 627 & 148 (24\%) & 67 (45\%) & $81(55 \%)$ \\
\hline \multicolumn{5}{|c|}{ Household owned at least one ITNs } \\
\hline Yes & 407 & $99(24 \%)$ & $46(46 \%)$ & $52(52 \%)$ \\
\hline No & 403 & $105(26 \%)$ & $46(44 \%)$ & $59(56 \%)$ \\
\hline \multicolumn{5}{|c|}{ Frequency of night slept under ITNs in the last 15 days } \\
\hline All nights & 188 & $45(24 \%)$ & $20(44 \%)$ & $24(53 \%)$ \\
\hline Almost all nights & 26 & $5(19 \%)$ & $3(60 \%)$ & $2(40 \%)$ \\
\hline Sometimes & 121 & $31(26 \%)$ & $13(42 \%)$ & $18(58 \%)$ \\
\hline Only few night & 8 & $2(25 \%)$ & $1(50 \%)$ & $1(50 \%)$ \\
\hline None of the nights & 64 & $16(25 \%)$ & $9(56 \%)$ & $7(44 \%)$ \\
\hline \multicolumn{5}{|c|}{ Sought treatment before visiting the health center } \\
\hline Yes & 75 & $18(24 \%)$ & $9(50 \%)$ & $9(50 \%)$ \\
\hline No & 735 & $186(25 \%)$ & $83(45 \%)$ & $102(55 \%)$ \\
\hline \multicolumn{5}{|c|}{ Number of days after illness onset } \\
\hline$\leq 2$ days & 140 & $27(19 \%)$ & $9(33 \%)$ & $18(67 \%)$ \\
\hline$>2$ days & 670 & 177 (26\%) & 83 (47\% & 93 (52\%) \\
\hline
\end{tabular}

One individual had infection with both Plasmodium falciparum and vivax

A significant number of $P$. falciparum cases occur in Ethiopia during the peak malaria transmission mainly in October. The national figure of $30 \%-40 \%$ of malaria cases in Ethiopia is due to P. vivax [10]. In contrast, in this study the prevalence of $P$. vivax is higher than $P$. falciparum. Likewise, $P$. vivax was the main causative agent of malaria in Oromia Regional State of Ethiopia, which accounted for $60 \%$ of slide-positive cases [3]. A study conducted in East Shewa indicated a proportion of
53\% for P. falciparum and $47 \%$ for P. vivax [17]. The higher proportion of $P$. vivax in our study is consistent with studies conducted in other parts of Ethiopia $[16,18-20]$, which indicates trend shift of species composition. Conversely, the dominance of $P$. falciparum was indicated by other studies conducted in different parts of Ethiopia [21-23]. This could be explained by the fact that the prevention and control activities of malaria in Ethiopia [20] mainly focus on $P$. falciparum as it is 
Table 4 Factors associated with test positivity for malaria

\begin{tabular}{|c|c|c|c|c|}
\hline \multirow[b]{2}{*}{ Variables } & \multicolumn{4}{|c|}{ Test positivity } \\
\hline & Negative & Positive & Crude OR (95\% Cl) & Adj. OR (95\% Cl) \\
\hline \multicolumn{5}{|l|}{ Health center } \\
\hline Modjo & 139 & 30 & 1 & 1 \\
\hline Meki & 105 & 70 & $3.1(1.9,5.1)$ & $4.1(2.4,7.1) * *$ \\
\hline Batu & 137 & 43 & $1.5(0.9,2.5)$ & $1.7(0.9,2.9)$ \\
\hline Bulbula & 128 & 19 & $0.7(0.4,1.3)$ & $0.6(0.3,1.2)$ \\
\hline Shashemene & 97 & 42 & $2.0(1.2,3.4)$ & $2.6(1.5,4.5)^{*}$ \\
\hline \multicolumn{5}{|l|}{ Residence } \\
\hline Rural & 238 & 96 & $1.4(0.9,1.9)$ & $1.7(1.1,2.6)^{*}$ \\
\hline Urban & 368 & 108 & 1 & 1 \\
\hline \multicolumn{5}{|l|}{ Sex } \\
\hline Female & 302 & 95 & $0.9(0.6,1.2)$ & $0.8(0.6,1.1)$ \\
\hline Male & 304 & 109 & 1 & 1 \\
\hline \multicolumn{5}{|l|}{ Age } \\
\hline $15-24$ & 220 & 93 & $6.0(1.9,15.6)$ & $6.7(2.3,19.5)^{*}$ \\
\hline $25-34$ & 224 & 75 & $4.4(1.5,12.4)$ & $4.2(1.4,12.4)^{*}$ \\
\hline $35-44$ & 110 & 32 & $3.8(1.3,11.3)$ & 3.nn $(1.2,11.4)^{*}$ \\
\hline$>45$ & 52 & 4 & 1 & 1 \\
\hline \multicolumn{5}{|l|}{ Type of roof } \\
\hline Thatched & 127 & 56 & $1.4(0.9,2.1)$ & $1.5(0.9,2.3)$ \\
\hline Corrugated iron & 479 & 148 & 1 & 1 \\
\hline \multicolumn{5}{|c|}{ Household ownership of ITNs } \\
\hline No & 298 & 105 & $1.1(0.8,1.5)$ & $0.9(0.6,1.3)$ \\
\hline Yes & 308 & 99 & 1 & 1 \\
\hline \multicolumn{5}{|c|}{ Sought treatment before visiting the health center } \\
\hline Yes & 57 & 18 & $0.9(0.5,1.6)$ & $0.6(0.4,1.2)$ \\
\hline No & 549 & 186 & 1 & 1 \\
\hline \multicolumn{5}{|c|}{ Number of days after illness onset } \\
\hline$\leq 2$ days & 269 & 84 & $0.9(0.6,1.2)$ & $1.3(0.9,1.9)$ \\
\hline$>2$ days & 337 & 120 & 1 & 1 \\
\hline
\end{tabular}

*Significance level of $<0.05$, * Significance level of $<0.001$

deadlier than $P$. vivax [24]. Other possible reasons might be climate variability or that $P$. vivax might have developed resistance for Chloroquine.

Appropriate utilization of ITNs is one of the key interventions for the prevention of malaria [3]. In the present study, $50 \%$ of households had at least one ITN. Similarly, according to a malaria indicator survey conducted in $2011,55 \%$ of households residing in malaria-prone areas of Ethiopia owned at least one mosquito net (of any type), and Oromia was found to have the lowest net ownership (44\%) [3]. It is estimated that $42 \%$ of households in Africa owned at least one ITN in mid-2010 [25]. Moreover a study conducted in Eastern Ethiopia indicated an ITN ownership of $62 \%$ [26]. To the contrary, a study conducted in malaria epidemic prone areas of Ethiopia indicated that the overall ITN distribution was $98 \%$ [27]. The difference for this high value compared to our data could be explained by the reason that the present study is not a household survey which might have underestimated it. On the other hand, $41 \%$ of households without a single ITN represent a public health concern which needs to be addressed. The mean possession of bed net of 1.82 per household reported in our study is consistent with the report (1.73 /household) from study conducted in Ghana [28]. However, it is by far higher than the findings of malaria indicator survey conducted in 2011 (mean 0.7 /household) [3]. The ITN utilization of our study is high as compared to the study conducted in Eastern Ethiopia (21.5\%) [26]. 
The use of representative samples with a high response rate of $97 \%$ is the strength of the present study, however it has some limitations. This study is a facility based survey. Therefore, it does not represent the situation in the whole population but it already provides reliable important data. Data collection relied on information given by the interviewees. Practices such as presence, type and use of ITN could not be verified by direct observation. Moreover, the diagnosis of malaria did not include PCR (Polymerase Chain Reaction). As this was a pragmatic study in a real-life rural environment, blood film was available to diagnose malaria infection, rather than rapid diagnostic testing which has a higher sensitivity [29]. On top of that, microscopic tests of malaria were done by the laboratory technicians in the different settings who didn't get training about the determination of test positivity which could have led to bias due to interpersonal variation. However, these details reflect the 'realworld' nature of our data, that were based on usual clinical practice, and do not necessarily invalidate our findings.

\section{Conclusions}

In conclusion, findings of this study indicate that malaria is an important public health problem among adults in East Shewa with the predominance of $P$. vivax and $P$. falciparum; and being in the productive age group, living in Meki or Shashemene, and living in rural areas, were risk factors for malaria infection. According to WHO when the slide positivity rate of all febrile patients with suspected malaria is less than $5 \%$, the country could consider transitioning into "pre-elimination" [11]. Therefore, a test positivity rate of $25 \%$ at health facility level indicates that malaria is a major burden in the zone, which is not in line with the national strategic plan for malaria prevention control and elimination in Ethiopia. Moreover, there is a gap regarding the mosquito nets/ ITNs ownership and utilization. Hence, more focus should be given to environmental sanitation as well as the consistent utilization of ITNs should be promoted by health workers and health extension workers in particular. In addition, the number of mosquito nets/ITNs supplied to households should be increased in order to assure adequate mosquito nets/ITNs ownership in each household. Further study using direct observation at sleeping time rather than reported use is important to assess ownership proper utilization of ITNs. Special attention should be given to those living in the rural area of the zone. Furthermore, there was an increased risk of malaria infection among the younger age group as well as among those living in Meki and Shashemene areas which needs a further investigation.

\section{Additional file}

Additional file 1: SPSS data set for the research entitled "Prevalence and associated risk factors of malaria among adults in East Shewa Zone of Oromia Regional State, Ethiopia: A cross-sectional study". (SAV 637 kb)

\section{Abbreviations}

IRS: Indoor Residual Spraying; ITN : Insecticide Treated Net; OPD: Outpatient Department; PCR: Polymerase Chain Reaction; WHO: World Health Organization

\section{Acknowledgements}

Our thanks go to the Addis Ababa University School of Public Health for supporting the study. We are grateful to the Oromia Regional Health Bureau, East Shewa Zone Health Department and respective District and Town Administration Health Offices for their support in facilitating the implementation of this study. Finally, we are very grateful for data collectors and study participants who willingly took part in this study. This study would not have been possible without their involvement. This work was supported by the University of Nottingham and Nottingham University Hospital Charity.

\section{Funding}

This work was funded by the University of Nottingham and Nottingham University Hospital Charity. The sponsors of the study had no role in study design, data collection, data analysis, data interpretation, or writing of the report. The corresponding author had full access to the data in the study and had full responsibility for the decision to submit.

Availability of data and materials

All data generated or analyzed during this study are included in this published article and its supplementary information files.

\section{Authors' contributions}

FT was involved in proposal writing, designed the study and participated in coordination, supervision and the overall implementation of the project, analyzed the data, drafted and finalized the manuscript. WD and AWF conceived the study and participated in all stages of the study and revision of the manuscript. AWF obtained funding for the study. All authors read and approved the final version of the manuscript.

\section{Ethics approval and consent to participate}

The study protocol was reviewed and approved by the Research and Ethics Committee of the School of Public Health at the College of Health Sciences of Addis Ababa University and University of Nottingham. Written informed consent was obtained from each participant and confidentiality was maintained. Lastly, information and education was given to the study participants with regard to malaria signs and symptoms, early diagnosis and adequate treatment, and its prevention methods.

Consent for publication

Not applicable.

\section{Competing interests}

The authors have declared that there are no competing interests.

\section{Publisher's Note}

Springer Nature remains neutral with regard to jurisdictional claims in published maps and institutional affiliations.

\section{Author details}

${ }^{1}$ School of Public Health, College of Health Sciences and Medicine, Jigjiga University, Jigjiga, Ethiopia. ${ }^{2}$ Division of Epidemiology and Public Health, University of Nottingham, Nottingham, UK. ${ }^{3}$ Department of Preventive Medicine, School of Public Health, College of Health Sciences, Addis Ababa University, Addis Ababa, Ethiopia. 
Received: 9 January 2017 Accepted: 6 July 2017

Published online: 17 July 2017

\section{References}

1. Gebreyesus AT, Deressa W, Witten KH, Getachew A, Seboxa T. Malaria. In: Berhane Y, Hailemariam D, Kloos H, editors. Epidemiology and ecology of health and disease in Ethiopia. Addis Ababa: Shama Books; 2006. p. 556-76.

2. Federal Ministry of Health $(\mathrm{FMOH})$. Malaria and Other Vector-borne Diseases Control Unit. Addis Ababa, Ethiopia: Federal Ministry of Health of Ethiopia; 1999.

3. Federal Ministry of Health $(\mathrm{FMOH})$ : Ethiopia National Malaria Indicator Survey 2011: Technical Summary Ethiopia: Ministry of Health of Ethiopia; 2012.

4. Federal Ministry of Health $(\mathrm{FMOH})$ : Health and health-related indicators, 2009/2010 Addis Ababa, Ethiopia: Ministry of Health of Ethiopia, 2011.

5. C-Change, FHI 360. Essential Malaria Actions: Ethiopia December 2012; 1-7.

6. World Malaria Report 2011: www.who.int/malaria/world_malaria_report_ 2011/en/

7. Federal Ministry of Health $(\mathrm{FMOH})$ : Ethiopia National Malaria Indicator Survey 2007. Addis Ababa: FMOH; 2008: 1-98.

8. Fontaine RE, Najjar AE, Prince JS. The 1958 malaria epidemic in Ethiopia. Am J Trop Med Hyg. 1961;10:795-803.

9. PMI. Malaria operational plan (MOP) Ethiopia. 2012:8-31.

10. Carter $C$. Annual malaria control program review enhancing impact through integrated strategies malaria programs Ethiopia and Nigeria. Atlanta: Georgia; 2012.

11. Federal Ministry of Health (FMOH). National strategic plan for malaria prevention, control and elimination in Ethiopia, 2011-2015. Addis Ababa, Ethiopia: 2010.

12. Tadesse F, Deressa W, Fogarty AW. Concerns about covert HIV testing are associated with delayed presentation in Ethiopian adults with suspected malaria: a cross-sectional study. BMC Public Health. 2016;16:102. doi:10.1186/s12889-016-2773-y.

13. Central Statistical Agency (CSA). The 2007 population and housing census of Ethiopia: Results for Oromia Regional State. Addis Ababa: 2007.

14. World Health Organization (WHO). A comparative study of three rapid diagnostic tests (RDTs) for malaria diagnosis in Oromia Regional State, Ethiopia. World Health Organization, Geneva, Switzerland, 2000.

15. Bekele S, Mengistu L, Abebe A, Daddi J, Girmay M, Berhanu E. Evaluation of the performance of CareStartTM malaria Pf/Pv combo and Paracheck PfR tests for the diagnosis of malaria in Wondo genet, southern Ethiopia. Acta Trop. 2009;111:321-4.

16. Alemu A, Abebe G, Tsegaye W, Golassa L. Climatic variables and malaria transmission dynamics in Jimma town. South West Ethiopia Parasit Vectors. 2011:4:30.

17. Deressa W, Chibsa S, Olana D. Treatment seeking of malaria patients in east Shewa zone of Oromia. Ethiop J Health Dev. 2003;17(1):9-16.

18. Tesfaye S, Belyhun Y, Teklu T, Mengesha T, Petros B. Malaria prevalence pattern observed in the highland fringe of Butajira, Southern Ethiopia: a longitudinal study from parasitological and entomological survey. Malar J. 2011;10:153

19. Woyessa A, Gebre-Michael T, Ali A. An indigenous malaria transmission in the outskirts of Addis Ababa, Akaki town and its environs. Ethiop J Health Dev. 2004;18:2-7.

20. Chala B, Petros B. Malaria in Finchaa sugar factory area in western Ethiopia: assessment of malaria as public health problem in Finchaa sugar factory based on clinical records and parasitological surveys, western Ethiopia. J Parasitol Vector Biology. 2011;3:52-8.

21. Ghebreyesus TA, Haile M, Witten KH, Getachew A, Yohannes M, Lindsay SW et al. Household risk factors for malaria among children in the Ethiopian highlands. Trans R Soc Trop Med Hyg. 2000;94:17-21.

22. Karunamoorthi K, Bekele M. Prevalence of malaria from peripheral blood smears examination: a 1-year retrospective study from the Serbo health center, Kersa Woreda. Ethiopia J Infect Public Health. 2009;2:171-6.

23. Ramos J, Reyes F, Tesfamariam A. Change in epidemiology of malaria infections in a rural area in Ethiopia. J Travel Med. 2005;12:155-6.

24. Baird J. Neglect of plasmodium vivax malaria. Trends Parasitol. 2007;23:533-9.

25. World Health Organization (WHO), Report WM. World Health Organization, Geneva. Switzerland. 2010;2010
26. Biadgilign S, Reda A, Kedir H. Determinants of ownership and utilization of insecticide-treated bed nets for malaria control in eastern Ethiopia. J Trop Med. 2012:1-7. doi:10.1155/2012/235015.

27. Animut A, Gebremichael T, Medhin G, Balkew M, Bashaye S, Seyoum A. Assessment of distribution, Knowledge and Utilization of Insecticide Treated Nets in Selected Malaria Prone Areas of Ethiopia. Ethiop J Health Dev. 2008;22(3):268-74

28. Adjei JK, Gyimah SO. Household bed net ownership and use in Ghana: implications for malaria control. Canadian Stud Popul. 2012;39:15-30.

29. William MS, Charles PC, Douglas AO, Billie AJ, Charlotte MT, Susan HB, et al. Diagnostic performance of rapid diagnostic tests versus blood smears for malaria in US clinical practice. Clin Infect Dis. 2009;49(6):908-13. doi:10.1086/605436.

\section{Submit your next manuscript to BioMed Central and we will help you at every step:}

- We accept pre-submission inquiries

- Our selector tool helps you to find the most relevant journal

- We provide round the clock customer support

- Convenient online submission

- Thorough peer review

- Inclusion in PubMed and all major indexing services

- Maximum visibility for your research

Submit your manuscript at www.biomedcentral.com/submit
) Biomed Central 